\title{
On the Design of a Diaphragm Valve for Aerostatic Bearings
}

\author{
Luigi Lentini ${ }^{1,}{ }^{*}$, Federico Colombo ${ }^{1}$, Andrea Trivella $^{1}$, Terenziano Raparelli ${ }^{1}$, Vladimir \\ Viktorov $^{1}$ \\ ${ }^{1}$ Politecnico di Torino, Corso Duca degli Abruzzi 24, 10129, Torino, Italy
}

\begin{abstract}
Because of their almost zero friction, cleanness and long life, aerostatic bearings are commonly used in many applications where high precision of positioning is required, e.g. machine tools, measuring machines, semiconductor manufacturing and power board testing. However, air bearings suffer from low relative stiffness and poor damping. Active and passive compensation are two effective methods to enhance the static and dynamic performance of these kinds of bearings. Despite their higher performance, active compensation solutions are too expensive to be used in industrial applications, as a consequence of the costs related to their controllers, actuators and sensors. This paper presents the design and performance of a passive compensation method that exploits a diaphragm valve. Thanks to its ease of integration, satisfactory performance and relatively low cost, this method could be a valuable solution to increasing the stiffness of aerostatic bearings. This work provides a procedure to design diaphragm valves depending on the type of the integrated pad and the desired nominal air gap height. Results demonstrate that, once correctly designed, the diaphragm valve makes it possible to obtain bearings with quasi-static infinite stiffness at the selected air gap height.
\end{abstract}

\section{Introduction}

Because of their almost zero friction, cleanness and long life, aerostatic bearings are commonly used in many applications where high precision of positioning is required, e.g. machine tools, measuring machines, semiconductor manufacturing and power board testing $[1,2]$.

However, because of the of the compressibility of the lubricant, air bearings suffer from low relative stiffness and poor damping. Since gas bearings were first used in industrial applications, many solutions have been proposed to reduce these limitations [1,2].

Firstly, the effect of the type, number, location and size of the supply holes was investigated numerically and experimentally. Kazimierski and Trojnarski [3] analysed the influence of the diameter of the supply holes on the load capacity and power consumption. Moreover, they considered the cases of bearings with pocketed and inherent orifices by proposing a general calculation method. Similarly, Boffey et al. [4] and Colombo et al. [5] performed experimental and numerical studies to investigate the effect of the supply pressure, orifice

* Corresponding author: luigi.lentini@polito.it 
size and location on the performance of rectangular aerostatic bearings. They found that it is possible to find an optimal distribution of the supply holes and that the optimal air gap height decreases as their diameters decrease. However, these works demonstrated that this kind of solution can only lead to modest enhancements of pad performance.

Machining shallow grooves or the use of porous restrictor represents two other early approaches that were used in order to obtain bearings with higher performance. Despite these approaches being more effective, they still presented some relevant drawbacks. In fact, the design of grooved pads is made more complex since the groove size has to be suitably selected to avoid pneumatic hammer $[6,7]$. Additionally, the presence of grooves in CFD, distributed [8] or lumped parameter models [9-11] notably increases the effort to accurately simulate the performance of these pads. At the same time, designing bearings with porous surfaces or restrictors [12] presents limitations related to the choice of materials that need to have suitable porosity [13] and impact toughness [14, 15].

Subsequently, many types of compensation methods have been proposed thanks to technological improvements, especially in the fields of manufacturing and electronics. These methods consist in the use of additional devices and/or elements to enhance aerostatic bearing performance, i.e., load capacity, stiffness, damping and air consumptions. Although the working principles of compensation methods can be the most varied, in general, they are aimed to compensate for air gap variations. The more common ways to obtain this compensation is to suitably modify the inherent characteristics of air bearing feeding system or/and actively change the bearing geometry. Moreover, compensation methods can be classified as passive or active [16]. Passive compensation methods use components whose functioning only requires the energy associated with the supply pressure of the bearing, e.g. pneumatic valves and compliant elements. Conversely, actively compensated bearings are equipped with elements that require external sources of energy in order to function, e.g. sensors, controllers and actuators. As demonstrated in many works, because of the presence of closed loop control systems, active compensation methods make it possible to obtain remarkable enhancements of the static and dynamic performance of gas bearings. Some of the most effective active compensation methods use actuators to suitably modify the shape of the bearing land, i.e. conicity control, or modify the bearing thickness, i.e. geometrical compensation. Some examples of active compensation methods that exploit conicity control are those provided by Al Bender [17] and Maamari et al. [18, 19]. Al Bender [17] proposed a protype of an active circular pad where three piezo actuators are used to actively control the conicity of the air gap geometry. Similarly, Maamari et al. [18, 19] proposed an active aerostatic bearing based on conicity control by means of magnetic actuation. Regarding the geometrical compensation method, Colombo et al. [20] developed an active pad where a piezo actuator, integrated with a custom-built compliant mechanism [21], is used to modify the bearing thickness.

Despite their limitations, the lower costs of passive compensation solutions may render them more attractive to be employed in industrial applications. Most of the current passive compensation solutions exploit low cost components such as elastic orifices [22], compliant surfaces [23], springs [24] or valves [25, 26]. It has been found that designing passive compensation systems exploiting diaphragm valves can lead to significant increases in stiffness [26] and damping in a low frequency range [27].

This paper presents a procedure to obtain an optimal design of diaphragm valves for aerostatic pads. To render the description of the method as simple as possible, the design procedure is developed by considering a circular aerostatic pad with a single inherent feed hole. Once the nominal air gap height at which the pad has to work has been selected, this procedure makes it possible to design a valve that can increase the stiffness of the bearing up to infinite stiffness. 


\section{Material and Methods}

\section{‥ 1 Air Pad Model}

Figure 1 shows the geometry of the circular pad considered in this analysis. The pad has a radius $R=20 \mathrm{~mm}$ and its supply hole has a radius $R_{0}=0.25 \mathrm{~mm} . p_{s}, p_{0}, p_{a}$ are the supply pressure of the pad, the pressure downstream from the restrictor and the ambient pressure, respectively. It is worth pointing out that the non-compensated pad can only work in the presence of a constant supply pressure $p_{s}=$ cost and that, depending on the pressure ratio $p_{0} / p_{s}$, the pad can work in subsonic $\left(p_{0} / p_{s} \geq b\right)$ or sonic conditions $\left(p_{0} / p_{s}<b\right)$.

On the basis of this, depending on the pressure ratio $p_{0} / p_{s}$, the air mass flow rate supplied to the air gap $G_{1}$ can be computed by considering the restrictor as an isoentropic nozzle which has a minimum cross section corresponding to the annular area of $2 \pi R_{0} h$ :

$$
\begin{gathered}
G_{1}=c_{d} \frac{p_{s}}{\sqrt{R_{g} T_{s}}} 2 \pi R_{0} h \sqrt{\frac{2 k}{k-1}\left[\left(\frac{p_{0}}{p_{s}}\right)^{\frac{2}{k}}-\left(\frac{p_{0}}{p_{s}}\right)^{\frac{k+1}{k}}\right]} \\
\frac{p_{0}}{p_{s}}>\left(\frac{2}{k+1}\right)^{\frac{k}{k-1}}=b \\
G_{1}=G_{c r}=c_{d} \frac{p_{s}}{\sqrt{R_{g} T_{s}}} 2 \pi R_{0} h_{c r} \sqrt{\frac{2 k}{k-1}\left[\left(\frac{2}{k+1}\right)^{\frac{2}{k-1}}-\left(\frac{2}{k+1}\right)^{\frac{k+1}{k-1}}\right]} \\
\frac{p_{0}}{p_{s}} \leq\left(\frac{2}{k+1}\right)^{\frac{k}{k-1}}=b
\end{gathered}
$$

where, $k$ and $R_{g}$ are the specific heat ratio and gas constant of the air. $T_{s}$ is the temperature of the supply air, $c_{d}$ is the discharge coefficient of the supply hole, $b=0.5283$ is the critical pressure ratio of the nozzle and $h_{c r}$ is the air gap height corresponding to the maximum air consumption of the pad $G_{c r}$.

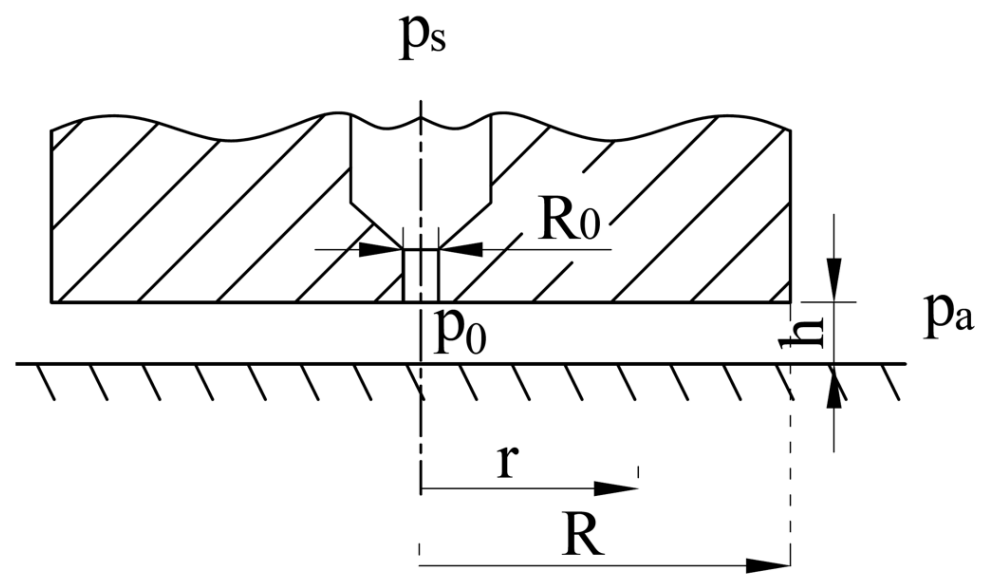

Fig. 1 Scheme of the circular air pad. 
The pressure distribution (see eq. 2) is obtained solving the one-dimensional Reynolds equation for the circular air pad considering as boundary conditions $\mathrm{p}=\mathrm{p}_{0}$ and $\mathrm{p}=\mathrm{p}_{\mathrm{a}}$ at inner and outer radius respectively. The load capacity of the pad $F_{p}$ can be computed by integrating the air gap pressure distribution $p(r)$.

$$
\begin{gathered}
p(r)=p_{0} \sqrt{1-\left[1-\left(\frac{p_{a}}{p_{0}}\right)^{2}\right] \frac{\ln \left(\frac{r}{R_{0}}\right)}{\ln \left(\frac{R}{R_{0}}\right)}} \\
F_{P}=p_{0} \pi R_{0}^{2} \sqrt{\frac{\pi A}{8}} e^{\frac{2}{A}}\left[\operatorname{erf}\left(\sqrt{\frac{2}{A}}\right)-\operatorname{erf}\left(\sqrt{\frac{2}{A}} \cdot \frac{p_{a}}{p_{0}}\right)\right] ; \quad A=\frac{\left(1-\frac{p_{a}^{2}}{p_{0}^{2}}\right)}{\ln \left(\frac{R}{R_{0}}\right)}
\end{gathered}
$$

The air mass flow rate exhausted from the gap $G_{2}$ can be computed as:

$$
\begin{gathered}
G_{2}=\frac{\pi h^{3}\left(p_{0}^{2}-p_{a}^{2}\right)}{12 \mu R_{g} T \cdot \ln \left(\frac{R}{R_{0}}\right)}, \quad \frac{p_{0}}{p_{s}}>\left(\frac{2}{k+1}\right)^{\frac{k}{k-1}}=b \\
G_{2}=G_{c r}, \quad \frac{p_{0}}{p_{s}} \leq\left(\frac{2}{k+1}\right)^{\frac{k}{k-1}}=b
\end{gathered}
$$

The characteristic curves of the pad are obtained by imposing different values of $p_{0}\left(p_{a}<\right.$ $\left.p_{0}<p_{s}\right)^{\dagger}$ and computing the corresponding air gap height by imposing the mass conservation $\left(G_{1}=G_{2}\right)$ :

$$
\begin{gathered}
h=\left\{\frac{12 \mu R_{g} T \cdot \ln \left(\frac{R}{R_{0}}\right)}{\left(p_{0}^{2}-p_{a}^{2}\right)} \cdot c_{d} \frac{p_{s}}{\sqrt{R_{g} T_{s}}} 2 R_{0} \sqrt{\frac{2 k}{k-1}\left[\left(\frac{p_{0}}{p_{s}}\right)^{\frac{2}{k}}-\left(\frac{p_{0}}{p_{s}}\right)^{\frac{k+1}{k}}\right]}\right\}^{1 / 2}, \\
\frac{p_{0}}{p_{s}}>\left(\frac{2}{k+1}\right)^{\frac{k}{k-1}}=b \\
h=\left\{\frac{G_{c r} 12 \mu R_{g} T \cdot \ln \left(\frac{R}{R_{0}}\right)}{\pi\left(p_{0}^{2}-p_{a}^{2}\right)} \cdot\right\}^{1 / 3}, \\
\frac{p_{0}}{p_{s}} \leq\left(\frac{2}{k+1}\right)^{\frac{k}{k-1}}=b
\end{gathered}
$$

$\dagger$ This choice is equivalent to defining an external load acting on the pad. 
Once the air gap height has been computed, the load capacity and the air consumption of the pad can be obtained through Equations 1, 3 and 4. Figures $2 \mathrm{a}$ and $2 \mathrm{~b}$ show the characteristic curves obtained in the presence of supply pressures ranging from 0.2 to 0.7 $\mathrm{MPa}$. The choice of this range is due to the fact that the maximum supply pressure usually used in industrial applications is about 0.7 absolute $\mathrm{MPa}$.

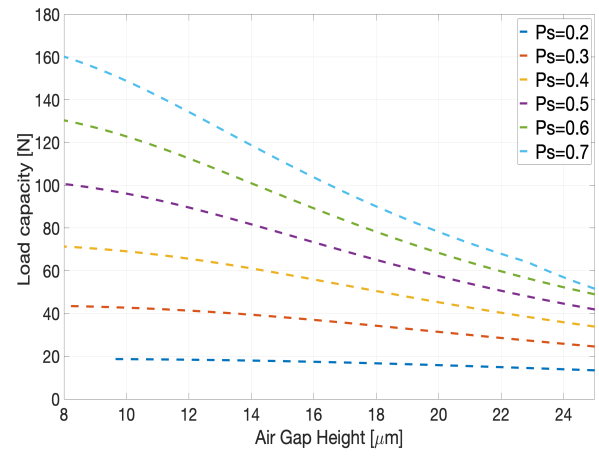

Fig. 2a Load Capacity of the circular pad in the presence of different $p_{s}$.

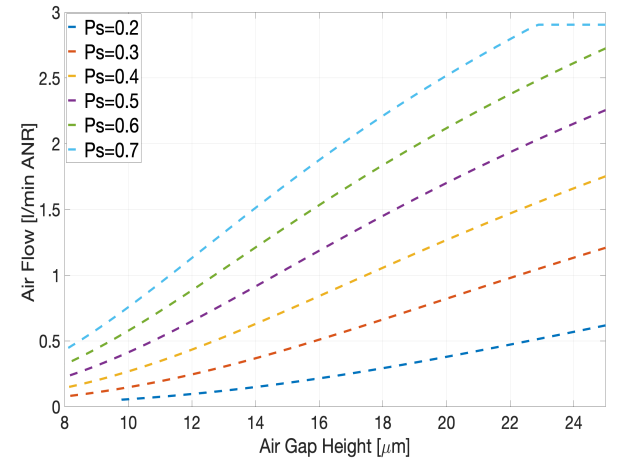

Fig. 2b Air consumption of the circular pad in the presence of different $p_{s}$.

\section{2 Diaphragm Valve Model}

Figure 3 shows a scheme of the valve illustrating its main functional dimensions and parameters. The valve consists in a compliant chamber supplied by a nozzle. The compliance of the chamber is due to the presence of a circular membrane of radius $R_{m}$ that deflects depending on the value of the pressure in the valve. When the valve is connected to the pad, this pressure corresponds to the supply pressure of the bearing $p_{s}$ (see Figure 1). During the functioning of the compensated pad, the values of $p_{s}$ can vary depending on the dimension of the nozzle duct (radius: $R_{v}$ ), the initial distance between the nozzle and the membrane $x_{0}$, the load applied to the pad $\left(F_{\text {ext }}\right)$ and the membrane stiffness $k_{m}$.

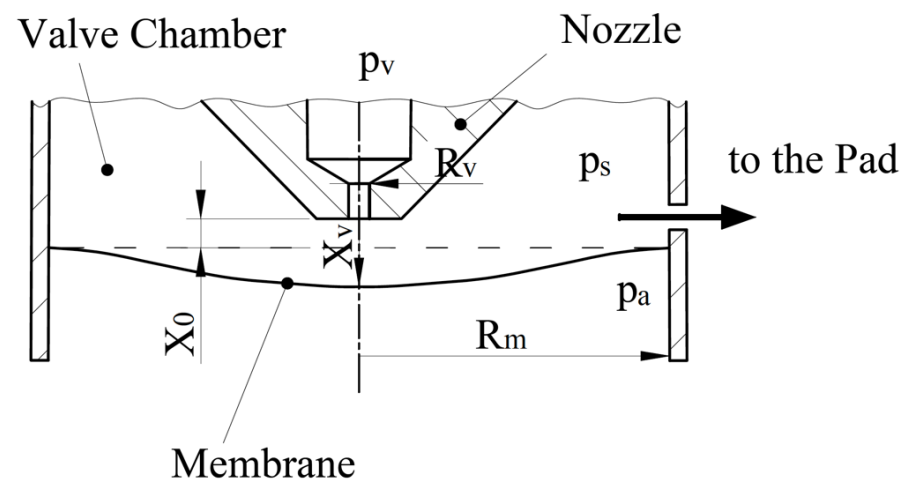

Fig. 3 Functional scheme of diaphragm valve.

As previously stated for the supply hole of the pad, the air mass flow rate provided by the nozzle $G_{v}$ of the valve is computed by considering the formula used for isoentropic nozzles: 


$$
\begin{gathered}
G_{v}=c_{d, v} \frac{p_{v}}{\sqrt{R_{g} T_{v}}} 2 \pi R_{v} x_{v} \sqrt{\frac{2 k}{k-1}\left[\left(\frac{p_{s}}{p_{v}}\right)^{\frac{2}{k}}-\left(\frac{p_{s}}{p_{v}}\right)^{\frac{k+1}{k}}\right]} \\
\frac{p_{s}}{p_{v}}>\left(\frac{2}{k+1}\right)^{\frac{k}{k-1}}=b \\
G_{v}=G_{v, c r}=c_{d, v} \frac{p_{v}}{\sqrt{R_{g} T_{v}}} 2 \pi R_{v} x_{v} \sqrt{\frac{2 k}{k-1}\left[\left(\frac{2}{k+1}\right)^{\frac{2}{k-1}}-\left(\frac{2}{k+1}\right)^{\frac{k+1}{k-1}}\right]} \\
\frac{p_{s}}{p_{v}} \leq\left(\frac{2}{k+1}\right)^{\frac{k}{k-1}}=b
\end{gathered}
$$

where, $T_{v}$ is the air temperature in the valve and $x_{v}$ is the distance between the nozzle and the membrane that is computed considering the membrane as a linear spring:

$$
\begin{gathered}
x_{v}=x_{0}+\frac{\pi R_{m}^{2}\left(p_{s}-p_{a}\right)}{k_{m}} ; x_{v}>x_{b y-p a s s} \\
x_{v}=x_{b y-p a s s} ; x_{v} \leq x_{b y-p a s s}
\end{gathered}
$$

where, $\mathrm{x}_{\mathrm{by} \text {-pass }}$ is a distance that takes into account the air leakages experienced when the load applied to the pad is low and the nozzle is used to preload the membrane $\left(\mathrm{x}_{0}<0\right)$. In these instances, pressurized air may flow into the valve chamber even if the nozzle is in contact with the membrane ${ }^{\ddagger}$ ). The reasons for these leakages can be attributed to different phenomena, e.g. the deflection of the membrane, a small inclination of the nozzle and the roughness of the mating surfaces.

\section{3 The Proposed Design Procedure}

The analysis of Equation 1 reveals that, given different values of the pressure downstream from the restrictor $p_{0}$, it is always possible to find the related values of $p_{s, i d}$ that provide a constant air gap height $h$. Moreover, from Equation 3, it can be seen that for each value of $p_{0}$ only one value of the external load $F_{p}=F_{\text {ext }}$ exists. These two observations imply that, in the presence of load variations, it is always possible to find an optimal supply pressure of the $\operatorname{pad} p_{s}$ that keeps the air gap height constant. In view of this, the main goal is to design the diaphragm valve in such a way that it could provide values of $p_{s}$ which are as close as possible to the optimal one. The proposed design procedure can easily be extended to every kind of aerostatic pad that can be modelled through a lumped parameter model.

\section{3.1 Research of the Optimal Values of $p_{s, i d}$}

Once the desired air gap height of the pad $h_{\text {des }}$ has been designed, the optimal values of $p_{s, i d}$ can be found by imposing different values of $p_{0}$ and iteratively solving the following equations:

† The pressure in the valve $p_{s}$ is not enough to overcome the pressure due to the preload. 


$$
\begin{gathered}
G_{2}-c_{d} \frac{p_{s, i d}}{\sqrt{R_{g} T_{s}}} \pi R_{0} h_{d e s} \sqrt{\frac{2 k}{k-1}\left[\left(\frac{p_{0}}{p_{s, i d}}\right)^{\frac{2}{k}}-\left(\frac{p_{0}}{p_{s, i d}}\right)^{\frac{k+1}{k}}\right]}=0, \\
\frac{p_{0}}{p_{s, i d}}>\left(\frac{2}{k+1}\right)^{\frac{k}{k-1}}=b \\
G_{2, c r}-c_{d} \frac{p_{s, i d}}{\sqrt{R_{g} T_{s}}} \pi R_{0} h_{d e s} \sqrt{\frac{2 k}{k-1}\left[\left(\frac{2}{k+1}\right)^{\frac{2}{k-1}}-\left(\frac{2}{k+1}\right)^{\frac{k+1}{k-1}}\right]}=0, \\
\frac{p_{0}}{p_{s, i d}} \leq\left(\frac{2}{k+1}\right)^{\frac{k}{k-1}}=b
\end{gathered}
$$

These equations represent the fact that the continuity equation is satisfied in the presence of the desired air gap height $h_{d e s}$. In this case, the equation was solved via the regula falsi method, considering as initial values $p_{s, 1}=p_{0}$ and $p_{s, 1}=p_{v}$. Once these values are found, it is necessary to impose the physical limits due to supply pressure that can be used $p_{s, p r o g}=$ $p_{s, i d} \leq p_{v}$. Hence, the designed valve will not be able to compensate for load variations that require supply pressures higher than $p_{v}$.

\section{3.2 Diaphragm Valve Design}

By knowing the ideal values of the supply pressures $p_{s, p r o g}$, that the valve can supply to the pad, the related values of the membrane nozzle distance $x_{v \text {,des }}$ can be computed.

$$
\begin{aligned}
& x_{v, \text { des }}=\frac{G_{\text {prog }} \sqrt{R_{g} T_{v}}}{2 \pi R_{v} P_{v} \sqrt{\frac{2 k}{k-1}\left[\left(\frac{p_{s}}{p_{v}}\right)^{\frac{2}{k}}-\left(\frac{p_{s}}{p_{v}}\right)^{\frac{k+1}{k}}\right.}}, \\
& \frac{p_{s}}{p_{v}}>\left(\frac{2}{k+1}\right)^{\frac{k}{k-1}}=b \\
& x_{v, \text { des }}=\frac{G_{\text {prog } \sqrt{R_{g} T_{v}}}}{2 \pi R_{v} P_{v} \sqrt{\frac{2 k}{k-1}\left[\left(\frac{2}{k+1}\right)^{\frac{2}{k-1}}-\left(\frac{2}{k+1}\right)^{\frac{k+1}{k-1}}\right.}} \\
& \frac{p_{s}}{p_{v}} \leq\left(\frac{2}{k+1}\right)^{\frac{k}{k-1}}=b
\end{aligned}
$$

where, $G_{\text {prog }}$ is the air mass flow rate of the pad computed using the pressure ratios $p_{0} / p_{s, p r o g}$ considered in the previous step. Figure 4 shows the trend of $x_{v \text {,des }}$ obtained for different ratios of the valve and the pad hole diameters $R_{v} / R_{0}$. 


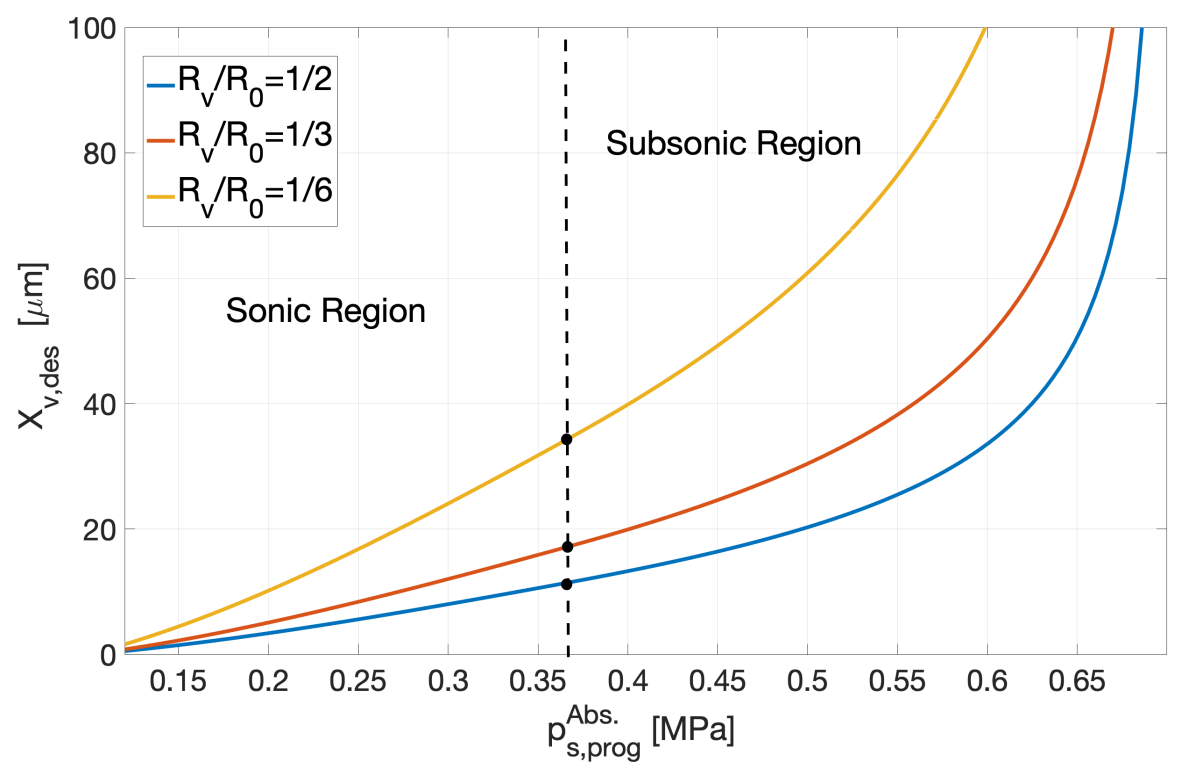

Fig. 4 Trend of $x_{v \text {,des }}$ obtained for different ratios of the valve and the pad hole diameters $R_{v} / R_{0}$.

This figure makes it possible to see that reducing the diameter of the nozzle requires a higher value of $x_{v}$ (more compliant membrane) and highly non-linear trends. In view of this, a better choice would be to design the diameter of the nozzle as big as possible. Regarding this choice, it is necessary to consider the fact that the air consumption of the compensated pad can be regulated by the valve or the supply hole of the pad, depending on which has the smallest cross-section:

$$
\begin{array}{ll}
2 \pi R_{0} h<2 \pi R_{v} x_{v} & \text { (Case } 1) \\
2 \pi R_{0} h>2 \pi R_{v} x_{v} & (\text { Case } 2)
\end{array}
$$

When the annular area under the supply hole of the pad is lower than that under the valve nozzle, the air consumption of the controlled pad is regulated through the air gap height (Case 1), whereas the opposite occurs when the annular area under the supply hole of the pad is larger than that under the valve nozzle. Since it is favorable that the behavior of the pad is controlled by the valve, to enlarge the range where the valve can compensate for load variations, $R_{v} / R_{0}$ was taken in the order of $1 / 2$. Additionally, in this way the portion of the curve that can be approximated as linear enlarges. After this, it is possible to define the radius, the preload and the stiffness of the membrane $\left(R_{m} x_{0}\right.$ and $\left.k_{m}\right)$. The radius $R_{m}$ has to be chosen depending on the space available close to the valve (in this case it was selected as equal to $3 \mathrm{~mm}$ ), whereas the choice of the preload and the stiffness requires further consideration of the functioning of the nozzle in subsonic and sonic conditions. As it is possible to see from Figure 4, the almost linear part of the curves corresponds to $p_{s, p r o g}$. where the nozzle is sonic. For this reason, the preload $x_{0}$ and the stiffness of the membrane $k_{m}$ can be obtained by approximating the curves as linear between two points that belong to the range where the nozzle is sonic: $p_{s 1, p r o g}=0.2 \mathrm{Mpa}$ and $p_{s 2, \operatorname{prog}}=b \cdot p_{v}$. 


$$
\begin{gathered}
x_{0}=x_{v, 1}-\left(\frac{x_{v, 2}-x_{v, 1}}{p_{s 2, \text { prog }}-p_{s 1, \text { prog }}}\right) \cdot p_{s 2, \text { prog }} \\
k_{m}=\frac{\pi R_{m}^{2}}{\left(\frac{x_{v, 2}-x_{v, 1}}{p_{s 2, p r o g}-p_{s 1, \text { prog }}}\right)}
\end{gathered}
$$

These two pressures define the three characteristic regions of the curve of the compensated pad: saturation, compensation and by-pass region. Table 1 reports the data related to the case considered:

Table 1. Data related to the case considered $\left(p_{s 1, p r o g}=0.2 \mathrm{Mpa}\right.$ and $\left.p_{s 2, \operatorname{prog}}=b \cdot p_{v}\right)$.

\begin{tabular}{|c|c|c|}
\hline $\boldsymbol{h}_{\boldsymbol{d e s}}[\boldsymbol{\mu \mathbf { m } ]}$ & $\boldsymbol{x}_{\mathbf{0}}[\boldsymbol{\mu} \mathbf{m}]$ & $\boldsymbol{k}_{\boldsymbol{m}}[\mathbf{N} / \mathbf{m}]$ \\
\hline 10 & -7.00 & $1.77 \mathrm{e}+06$ \\
\hline 15 & -1.41 & $6.62 \mathrm{e}+05$ \\
\hline 20 & -1.01 & $4.05 \mathrm{e}+05$ \\
\hline
\end{tabular}

Figure 5 shows a scheme summarizing the steps of the proposed design procedure.

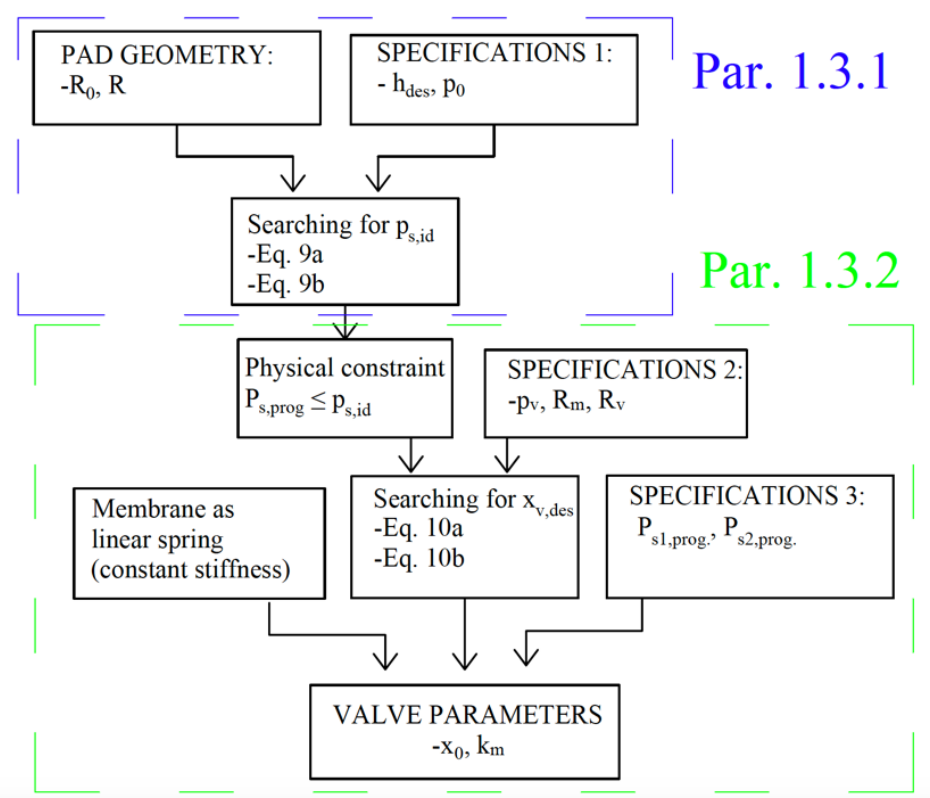

Fig. 5 Scheme summarizing the steps of the proposed procedure.

\section{Results and Discussions}

Figure 6 shows the load capacity of the compensated pad considering a compensation region between $p_{s 1, \operatorname{prog}}=0.2 \mathrm{MPa}$ and $p_{s 2 \text {,prog }}=b \cdot p_{v}$, and three different values of the desired air gap height $h_{\text {des }}=10,15$ and $20 \mu \mathrm{m}$. Moreover, to make the behavior of the 
compensated pad more evident, Figure 6 also shows the curve of the considered pad (not compensated) in the presence of supply pressures $p_{s, p a d}=p_{s 1, p r o g}, p_{s 2 \text {,prog }}$ and $p_{v}$. As can be seen, all the curves present a similar trend: starting from higher air gap heights they present a by-pass $\left(A_{h_{\text {des }}}-B_{h_{\text {des }}}\right.$, compensation $\left(B_{h_{\text {des }}}-C_{h_{\text {des }}}\right.$ and saturation region $\left(C_{h_{\text {des }}}-D_{h_{\text {des }}}\right.$. In the by-pass region $\left(p_{s} \leq p_{s 1, p r o g}\right)$, the compensated pad behaves like constant pneumatic resistance, since the distance between the nozzle and the membrane of the valve is very low $\left(x_{v} \leq x_{\text {by-pass }}\right)$, because of the initial preload applied to the membrane $x_{0}$ (see Table 1). As the external load applied to the pad increases, the pressure inside the chamber of the valve $p_{s}$ increases, too. When $p_{s}$ makes it possible to overcome the preload applied on the membrane $\left(p_{s}=p_{s 1, p r o g}=0.2 \mathrm{Mpa}\right)$, the valve starts compensating $\left(B_{h_{d e s}}\right)$. By looking at the compensation regions obtained, it is possible to see that, when the desired air gap height is $h_{d e s}=20 \mu \mathrm{m}$, the slope of the curve is almost vertical, whereas the curves related to $h_{d e s}=15$ and $10 \mu \mathrm{m}$ exhibit a variation from negative to positive values. This means that the behavior of the valve designed differs from the ideal one the more the value of the desired air gap height is reduced. However, it is possible to see that the variations of the air gap in the compensation regions are negligible in all the cases presented.

The saturation region begins when the $p_{s}$ is greater than $p_{s 2, p r o g}=0.3681 \mathrm{MPa}$. Here, the behavior of the compensated pad tends asymptotically to that of the not compensated one in the presence of a supply pressure equal to that of the valve $p_{s, p a d}=p_{v}$.

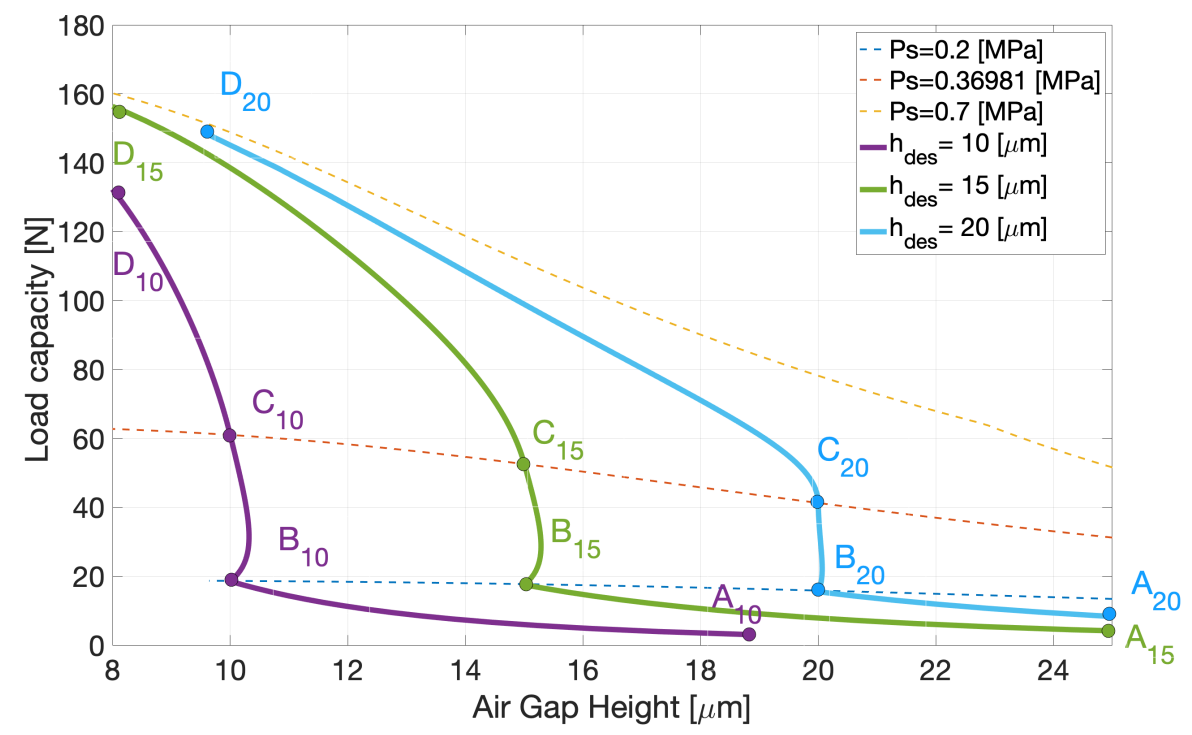

Fig. 6 The load capacity of the compensated pad considering different values of the desired air gap height $h_{d e s}=10,15$ and $20 \mu \mathrm{m}$.

\section{Conclusions}

This paper has presented a straightforward procedure to design diaphragm valves for aerostatic pads. As described in previous works [25-27], these kinds of valves have previously been employed in passive compensation methods aimed at increasing air pad performance. For the sake of simplicity, the proposed design procedure was described by considering a centrally fed circular pad with an inherent orifice. It was shown that it is 
possible to suitably design diaphragm valves on the basis of the features of the pad considered and the air gap height at which it has to work. Results showed that the controlled pad presents almost infinite stiffness on the selected compensation region. In view of this, and the fact that this kind of compensation method makes it possible to also increase damping significantly, this method would be a cost-effective solution that could be used in many applications.

\section{References}

1. L. Lentini, M. Moradi, and F. Colombo, Tribology in Industry Vol. 40, No. 2, pp. 165182, (2018).

2. Q. Gao, W. Chen, L. Lu, D. Huo, and K. Cheng, Tribology International Vol. 135, pp. 117. (2019).

3. Z. Kazimierski and J. Trojnarski, Journal of Lubrication Technology Vol. 102, pp 59-64 (1980).

4. D. A. Boffey, A. E. Duncan, and J. K. Dearden, Tribology International Vol. 14(5), pp 287-291 (1981).

5. F. Colombo, L. Lentini, T. Raparelli, A. Trivella, and V. Viktorov, Tribology Letters Vol. 66(4), 133 (2018).

6. M. Arghir, M.-A. Hassini, F. Balducchi, and R. Gauthier, Journal of Engineering for Gas Turbines and Power Vol. 138(2), (2016).

7. T. B. Stowell, Journal of Tribology Vol. 93, pp. 498-503 (1971).

8. M. F. Chen and Y. T. Lin, Tribology International Vol. 35(5), pp. 329-338 (2002).

9. F. Colombo, L. Lentini, T. Raparelli, A. Trivella, and V. Viktorov, Mechanisms and Machine Science, Vol. 67, pp. 678-686, (2019).

10. F. Colombo, L. Lentini, T. Raparelli, A. Trivella, and V. Viktorov, Mechanisms and Machine Science, Vol. 68, pp. 490-497, (2019).

11. A. van Beek and R. A. J. van Ostayen, Tribology International Vol. 39(8), pp. 833-838 (2006).

12. T. S. Luong, W. Potze, J. B. Post, R. A. J. Van Ostayen, and A. Van Beek, Tribology International Vol. 37(10), pp. 825-832 (2004).

13. G. Belforte, T. Raparelli, V. Viktorov, and A. Trivella, Tribology International Vol. 42(4), pp. 600-608 (2009).

14. M. Fourka and M. Bonis, Wear Vol. 210(1-2), pp. 311-317 (1997).

15. Y. B. P. Kwan and J. Corbett, Wear Vol. 222(2), pp. 69-73 (1998).

16. T. Raparelli, V. Viktorov, F. Colombo, and L. Lentini, Precision Engineering Vol.44, pp. 1-12(2016).

17. F. Al-Bender, Precision Engineering Vol. 33(2), pp.117-126 (2009).

18. N. Maamari, A. Krebs, S. Weikert, and K. Wegener, Tribology International Vol. 129, pp. 297-313 (2019).

19. N. Maamari, A. Krebs, S. Weikert, H. Wild, and K. Wegener, Tribology International Vol. 138, pp. 279-296 (2019).

20. F. Colombo, L. Lentini, T. Raparelli, and V. Viktorov, Meccanica, Vol. 52(15), pp. 3645-3660 (2017).

21. F. Colombo, L. Lentini, T. Raparelli, and V. Viktorov, International Journal of Applied Engineering Research Vol. 12(18), pp. $7803-7815$ (2017).

22. P. M. Newgard and R. L. Kiang, A S L E Transactions Vol. 9(3), pp. 311-317 (1966).

23. P. L. Holster and J. A. H. Jacobs, Tribology International Vol. 20(5), pp. 276-289 (1987).

24. M.-F. Chen and Y.-T. Lin, JSME International Journal Series C Vol. 45(2), pp. 492-501 (2002). 
25. D. Ghodsiyeh, F. Colombo, T. Raparelli, A. Trivella, and V. Viktorov, Tribology International Vol. 109, pp. 328-335 (2017).

26. D. Ghodsiyeh, F. Colombo, L. Lentini, T. Raparelli, A. Trivella, and V. Viktorov, Tribology International Vol. 141, 105964 (2020).

27. F. Colombo, L. Lentini, T. Raparelli, A. Trivella, and V. Viktorov (Under Press), Mechanisms and Machine Science, Vol. ??, pp. ???-???, (2020). 